\title{
Expression of A disintegrin and metalloprotease 8 is associated with cell growth and poor survival in colorectal cancer
}

Zuli Yang ${ }^{1 \dagger}$, Yang Bai ${ }^{1 \dagger}$, Lijun Huo ${ }^{2}$, Hao Chen ${ }^{1}$, Jintuan Huang ${ }^{1}$, Jizheng $\mathrm{Li}^{1}$, Xinjuan Fan ${ }^{3}$ Zihuan Yang ${ }^{3}$, Lei Wang ${ }^{4^{*}}$ and Jianping Wang ${ }^{4^{*}}$

\begin{abstract}
Background: A disintegrin and metalloprotease 8 (ADAM8) has been reported to be associated with various malignancies. However, no studies have examined ADAM8 association in colorectal cancer (CRC). The aim of this study was to investigate the expression and function of ADAM8 in CRC.

Methods: Expression level of ADAM8 in CRC was evaluated by quantitative RT-PCR, western blot and immunohistochemical staining analysis. The role of ADAM8 in colorectal carcinogenesis was evaluated by in vitro assays. The correlations between ADAM8 status and clinicopathological features including survival were analyzed.

Results: ADAM8 was highly expressed in CRC tissues compared with adjacent normal tissues. Knockdown of ADAM8 in two CRC cell lines resulted in reduced cellular growth and proliferation, and increased apoptosis.

Immunohistochemistry analysis showed no significant correlations of ADAM8 protein expression with clinicopathologic features. Survival analysis indicated that patients with ADAM8-positive tumors had worse 5 -year overall survival (OS, $p=0.037)$ and 5 -year disease free survival (DFS, $p=0.014)$ compared with those with ADAM8-negative tumors. Multivariate analysis indicated ADAM8 expression was an independent prognostic factor for both OS and DFS (both $p<0.001$ ). Subgroup analysis showed that 5-year OS of colon cancer, T3-T4 stage and N0 stage was worse for patients with ADAM8-positive tumors than those with ADAM8-negative tumors $(p<0.05)$. The 5-year DFS in colon cancer, T3-T4 stage, N0 stage, TNM stage II, adenocarcinoma, moderate differentiation and male patient subgroups was also worse for patients with ADAM8-positive tumors than those with ADAM8-negative tumors $(p<0.05)$.
\end{abstract}

Conclusions: Our results show that ADAM8 is overexpressed in CRC, promotes cell growth and correlates with worse OS and DFS, and thus could serve as a biomarker for individual CRC patient therapy.

Keywords: Colorectal cancer, A disintegrin and metalloprotease 8 (ADAM8), Proliferation, Prognosis, Overall survival, Disease free survival

\section{Background}

Colorectal cancer (CRC) is the third most common cancer and the fifth leading cause of cancer-related deaths, with approximately 715,000 new cases and 70,000 deaths annually in China. The survival of CRC patients is closely correlated with conventional and clinicopathological

\footnotetext{
* Correspondence: leiwangyinghu@hotmail.com; wangjply01@sohu.com ${ }^{\dagger}$ Equal contributors

${ }^{4}$ Department of Colon \& Rectum Surgery, The Sixth Affiliated Hospital of Sun Yat-sen University (Guangdong Gastrointestinal and Anal Hospital), Sun Yat-Sen University Guangzhou, 26 YuancunErheng Road, Guangzhou 510655, P.R China

Full list of author information is available at the end of the article
}

characteristics, such as tumor location, differentiation grade and TNM stages [1]. However, in practice, CRC patients with the same pathological features may have different prognosis. To discover new treatment options and more precise assessment of this malignancy, some potential therapy targets and candidate biomarkers have been reported, such as adenomatous polyposis coli (APC) gene, K-RAS gene, p53 gene and microsatellite instability (MSI) [2]. Among these targets and candidate biomarkers, some are used to justify whether adjuvant therapy is suitable for individual CRC patients, including MSI and wild-type or mutation of K-RAS and BRAF in certain exons [3-5]. 
A disintegrin and metalloprotease 8 (ADAM8) is a member of the human ADAM family, which contains disintegrin and metalloprotease domains [6]. ADAM proteins are involved in cell adhesion, cell migration, cell fusion, membrane protein shedding and proteolysis $[7,8]$. Aberrant expression of ADAM8 has been identified in solid tumors, such as gliomas, lung cancer [9], pancreatic cancer [10], renal cell carcinomas [11] and prostate carcinomas [12]. ADAM8 overexpression has been associated with poor prognosis in hepatocellular carcinoma [13], breast cancer [14] and pancreatic adenocarcinoma [10], and might act as a potential therapeutic target. Mechanistically, ADAM8 is involved in tumorigenesis by stimulating angiogenesis $[14,15]$, increasing cellular abilities of invasion and migration $[10,14]$, and inhibiting cancer cell apoptosis [16]. Previous studies showed that ADAM9 [17], ADAM10 [18], ADAM17 [19], ADAM23 [20] and ADAM29 [21] were involved in colorectal tumorigenesis and that ADAM8 was involved in lymph node metastasis of gastric cancer. However, the possible role of ADAM8 in CRC has not yet been evaluated. In the present study, we report the identification of ADAM8 as a novel biomarker and a potential prognostic indicator, and also provide evidence for its possible role in human colorectal carcinogenesis.

\section{Methods}

\section{Tissue samples, cell culture and cDNA preparation}

Thirty CRC tissue samples sets (each containing tumor and adjacent tissues) were obtained from the Sixth Affiliated Hospital of Sun Yat-sen University. Adjacent normal tissues were obtained at a distance of more than $5 \mathrm{~cm}$ from the tumor margin and confirmed by a pathologist. Eight human colorectal adenocarcinoma cell lines (HCT8, HT29, SW620, SW480, DLD1, HCT116, LOVO and CACO2) were purchased from the Culture Collection of Chinese Academy of Science (Shanghai, China), and cultured in RPMI 1640 supplemented with 10\% fetal bovine serum (Hyclone, USA) and $1 \%$ penicillin-streptomycin at $37^{\circ} \mathrm{C}$ in a $5 \% \mathrm{CO}_{2}$ incubator.

Total RNA from human CRC tissues cells was prepared using Trizol reagent (Invitrogen, Carlsbad, CA). Reverse transcription was performed using the ReverTra Ace qPCR RT Kit (TOYOBO CO., Osaka, Japan) according to the manufacturer's instructions.

This study was approved by the institutional review boards of Sun Yat-Sen University (Guangzhou, China), and written informed consent was obtained from each patient in this study.

\section{Tissue microarray (TMA)}

Three hundred and forty-two CRC samples were obtained from the tumor bank of the Department of Pathology of Sun Yat-Sen University (Guangzhou, China). The patients underwent initial surgical resection for CRC between January 2000 and November 2006 and were followed up until April 2010 to collect general information, pathology reports, and information regarding the patients' conditions after surgery. The samples were formalin-fixed and paraffin-embedded.

TMAs were constructed using an automated TMA instrument (ALPHELYS, Plaisir, France). After identifying the hematoxylin and eosin (H\&E)-stained slides for optimal tumor tissue, two cylindrical core biopsies (1 mm diameter) were punched from each formalin-fixed, paraffin-embedded tissue block and arrayed in recipient TMA blocks $(2 \times 3 \mathrm{~cm})$ as previously described [22].

\section{RNA interference (RNAi)}

ADAM8 siRNA oligonucleotides (si-ADAM8-1 sense 5'-GGACAAGCUAUAUCAGAAAdTdT-3' and antisense 3'-dTdTCCUGUUCGAUAUAGUCUUU-5'; and si-ADAM82 sense 5'-GCACCUGCAUGACAACGUAdTdT-3' and antisense 3'-dTdTCGUGGACGUACUGUUGCAU-5') and siRNA control oligonucleotides were obtained from RiboBio Co. Ltd (Guangzhou, China). HT29 and SW480 cells $\left(1 \times 10^{5}\right)$ were cultured in six-well plates until 50\% confluence and transfected with $100 \mathrm{nM}$ of the indicated siRNA using LipofectamineImax (Invitrogen, CA, USA) according to the manufacturer's instructions. The effects of siRNA silencing were analyzed after $48 \mathrm{~h}$ transfection. All experiments were repeated three times.

Quantitative real-time polymerase chain reaction (qRT-PCR) PCR was performed with each reaction containing $50 \mathrm{ng}$ of reverse-transcribed RNA and $1 \mu \mathrm{M}$ 5' and 3' primers in a $20 \mu \mathrm{L}$ reaction. The primers used are listed in Table 1. The reaction was performed on an ABI 7500 real-time PCR machine (Applied Biosystems, Foster City, CA, USA) using the following conditions: $95^{\circ} \mathrm{C}$ for $2 \mathrm{~min}, 40$ cycles of $95^{\circ} \mathrm{C}$ for $15 \mathrm{sec}$, and $60^{\circ} \mathrm{C}$ for $1 \mathrm{~min}$. Briefly, the relative RNA levels in each sample were determined by performing standard curves. $\beta$-actin levels were used for normalization.

\section{Immunohistochemistry (IHC) staining}

IHC was performed using the Polink-2 plus ${ }^{\oplus}$ Polymer HRP Detection System (GBI, USA) according to the manufacturer's instructions. After deparaffinization in xylene and rehydration through a graded alcohol series, slides were transferred to sodium citrate buffer (Beijing DingguoChangsheng Biotech Co. Ltd, AR-0511) for $15 \mathrm{~min}$ in the microware and left at room temperature for $30 \mathrm{~min}$. After blocking endogenous peroxidase, slides were incubated with $10 \mu \mathrm{g} / \mathrm{ml}$ goat polyclonal antibody specific to human ADAM8 (R\&D Systems, Inc., Minneapolis, $\mathrm{MN}$ ) at $4^{\circ} \mathrm{C}$ overnight. Slides were washed three times with phosphate-buffered saline (PBS) 
Table 1 Primers used for qRT-PCR

\begin{tabular}{lll}
\hline Name & Primer sequence forward & Primer sequence reverse \\
\hline ADAM8 & 5'-ACAATGCAGAGTTCCAGATGC-3' $^{\prime}$ & 5'-GGACCACACGGAAGTTGATT-3' $^{\prime}$ \\
-actin & 5'-CAATGAGCTGCGTGTGGCT-3' $^{\prime}$ & 5'-TAGCACAGCCTGGATAGC AA-3' $^{\prime}$ \\
\hline
\end{tabular}

and incubated with Polymer Helper (reagent 1, Polink-2

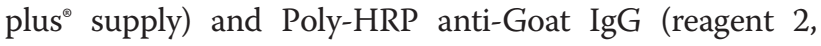
Polink-2 plus $^{\circ}$ supply) for $30 \mathrm{~min}$. Then the slides were stained with $\mathrm{DAB}$ and counterstained with hematoxylin. A negative control using antibody dilution as a substitute for primary antibody was performed for each experiment.

ADAM8 staining was examined by two pathologists blinded to clinicopathological data. Representative fields were captured under low power $(100 \times$ magnification $)$ and high power $(400 \times$ magnification $)$ by a Leica DMI 4000B inverted microscope (Leica Micro-systems, Wetzlar, Germany). Disagreements were reevaluated until a consensus was reached. IHC staining was analyzed using the Image Pro-Plus (version 6.0, Media Cybernetics, Silver Spring, USA) introduced by Xavier [23]. Briefly, the tumor area was selected as the area of interest (AOI), and the area sum and integrated optical density (IOD) of the AOI were selected as the measurement parameters. ADAM8 expression index equaled the quotient between the IOD and the total area of AOI. The mean expression index for each duplicate was used for statistical analysis. Selection of cutoff value was performed according to a previous study [24]. The cutoff point was 9.79 based on the patient's OS and DFS reaching significant difference. The CRC tissues were classified based on ADAM8 density into the negative group (less than or equal to 9.79) or positive group (more than 9.79). The ADAM8 positive group in cancer tissues and normal tissues was divided into three subgroups of weak (9.79-64.5), moderate (64.5-111.2) and strong (111.2-256.7) expression according to the IHC scores based on OS and DFD reaching significant difference.

\section{Western blot}

After $72 \mathrm{~h}$ transfection, HT29 and SW480 cells were washed three times with PBS and lysed with RIPA buffer (Dingguo, Beijing, China) supplemented with phenylmethanesulfonyl fluoride (PMSF, Dingguo, Beijing, China). ADAM8 protein levels were determined using two-color fluorescent western blotting on the Odyssey infrared imaging system (LI-COR, Nebraska, USA). In brief, protein samples were separated by $10 \%$ sodium dodecyl sulfatepolyacrylamide gel electrophoresis (SDS-PAGE) and transferred to a polyvinylidene fluoride (PVDF) membrane (Pall, New York, USA). Membranes were then blocked with $5 \%$ skim milk for $1 \mathrm{~h}$. Proteins were detected using mouse monoclonal antibodies specific to human ADAM8 (diluted 1:250, Abcam, UK, ab89127) and $\beta$-actin (diluted 1:10,000, Protein Tech, Chicago, USA). After incubating with primary antibodies overnight at $4{ }^{\circ} \mathrm{C}$ and species-appropriate fluorescently conjugated secondary antibodies for $1 \mathrm{~h}$ at room temperature, the blots were observed using the Odyssey infrared imaging system. Secondary antibodies were purchased from Santa Cruz Biotechnology (CA, USA) unless otherwise indicated.

\section{Cell viability and cell proliferation assay}

HT29 and SW480 cells were seeded in 96-well plates at a density of $1 \times 10^{4}$ cells/well. Cells were transfected with ADAM8 siRNA and cell viability was determined 0 , 1, 2, 3, and 4 days later using the CellTiter 96 Aqueous One Solution Cell Proliferation Assay kit (Promega, Madison, WI) according to the manufacturer's protocol. After $72 \mathrm{~h}$ of transfection with ADAM8 siRNA, cell proliferation assay was performed using an EDU (5-ethynyl-2'deoxyuridine) Cell Proliferation Kit (Invitrogen, Camarillo, $\mathrm{CA}$ ) according to the manufacturer's instructions. Data are presented as mean \pm SD for three independent experiments compared with the control group, and each experiment was performed in triplicate.

\section{Cell apoptosis assay}

HT29 and SW480 cells transfected with ADAM8 siRNA were seeded in 12 -well plates at a density of $1 \times 10^{4}$ viable cells/well. After $72 \mathrm{~h}$ culture, the cells were fixed in $70 \%$ ethanol and stained with $50 \mathrm{mg} / \mathrm{ml}$ propidium iodide (BD Pharmingen, San Jose, CA, USA), then sorted by FACSCalibur (BD Biosciences, Franklin Lakes, NJ, USA). Cell cycle profiles were analyzed by ModFit 3.0 software (Verity Software House, Topsham, ME, USA). Apoptosis was determined by dual staining with Annexin V:FITC and propidium iodide (Invitrogen). The Annexin V-positive cells were counted as apoptotic cells.

\section{Statistical analyses}

SPSS 16.0 for Windows (SPSS, Inc., Chicago, IL) was used for statistical analyses. Continuous variables were expressed as mean \pm SD and analyzed by t-test. The Chi-square test was used to show differences of categorical variables. Survival analysis was performed using the Kaplan-Meier method and compared using the log-rank test. $P<0.05$ was considered statistically significant. 


\section{Results}

Expression status of ADAM8 gene in human CRC tissues and cell lines

We evaluated the expression of ADAM8 protein and mRNA levels in 30 pairs of fresh-frozen CRC tissues and adjacent normal tissues by IHC and qRT-PCR. IHC

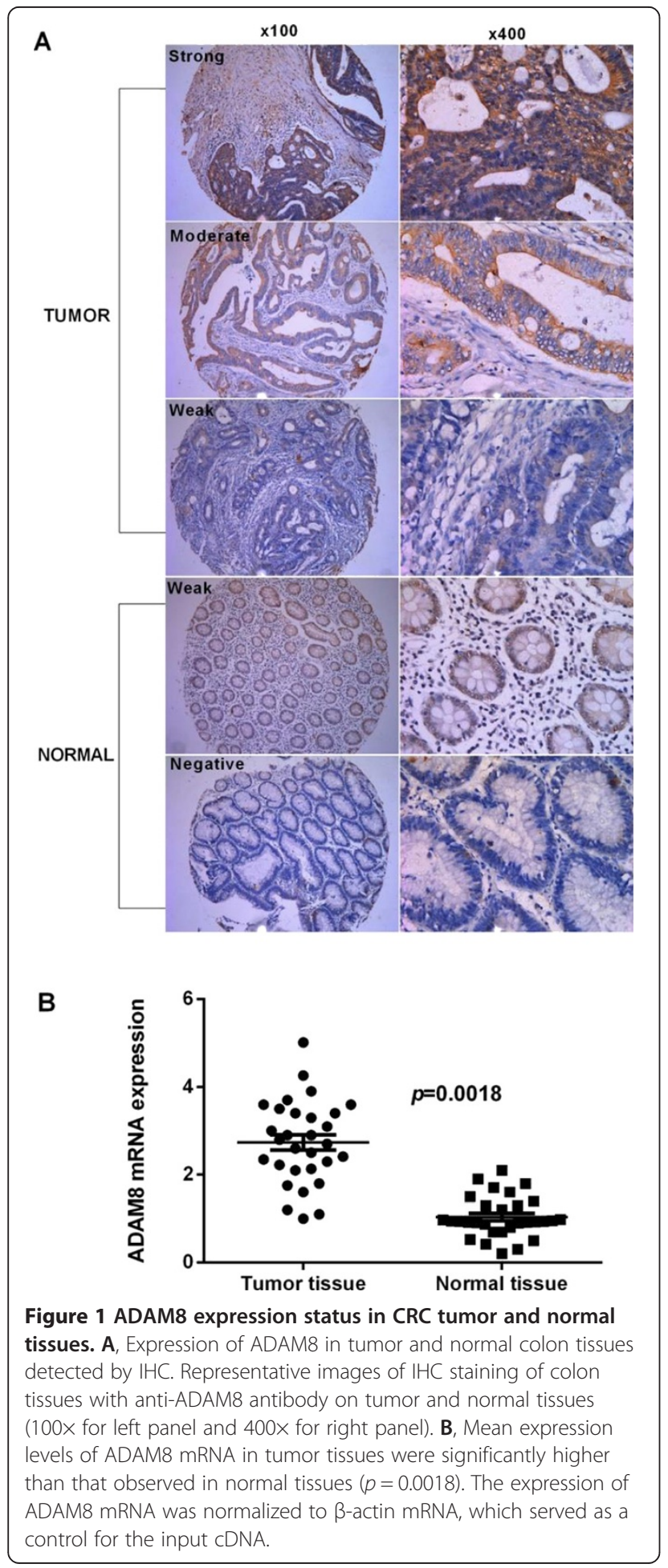

results showed that specific ADAM8 staining was mainly detected in the cytoplasm and membrane of noncancerous and malignant epithelial cells. IHC staining indicated more CRC tissues with positive ADAM8 expression than in corresponding adjacent normal tissues (81.0\% vs. 33.3\%, respectively; $p<0.0001$ ) (Figure $1 \mathrm{~A}$, Table 2 ). Among the 24 CRC patients with ADAM8 positive tumor tissues, high and moderate expression of ADAM8 was detected in 20 cases and weak expression in 4 cases. Among paired adjacent normal tissues, weak expression of ADAM8 protein was found in 10 cases, while no cases showed high or moderate expression. The mRNA expression levels of ADAM8 were evaluated by qRT-PCR and representative data are shown in Figures $1 \mathrm{~B}$ and $1 \mathrm{C}$. The mean expression levels of ADAM8 mRNA were significantly higher in tumor tissues compared with those in adjacent normal tissues $(2.74 \pm 0.17$ vs. $1.04 \pm 0.09$, respectively; $p=0.0018)$. The expression of ADAM8 mRNA was normalized to $\beta$-actin mRNA, which served as a control for the input cDNA.

Expression levels of ADAM8 mRNA and protein were also measured in eight CRC cell lines (Figure 2A). The expression level of ADAM8 protein was consistent with mRNA expression level in HCT8, HT29, SW620, SW480, HCT 116 and CACO2 cell lines, but not in DLD1 and LOVO cell lines. Based on these results, we selected HT29 and SW480 cell lines for further analysis. To explore the potential effect of ADAM8 on CRC carcinogenesis, two ADAM8 siRNA oligonucleotides were generated to knockdown ADAM8 expression in HT29 and SW480 cells. Transfection with si-ADAM8-1 decreased ADAM8 mRNA expression levels by $84.3 \%$ in HT29 cells $(p<0.001)$ and by $82.7 \%$ in SW480 cells $(p<0.001)$ compared with control siRNA. Transfection with si-ADAM8-2 decreased ADAM8 mRNA levels by $82.7 \%$ in HT29 cells $(p<0.001)$ and by $78.8 \%$ in SW480 cells $(p<0.001)$ (Figure $2 \mathrm{~B}$ and $\mathrm{C}$, top panel). Western blot analysis confirmed the qRT-PCR results (Figure $2 \mathrm{~B}$ and $\mathrm{C}$, bottom panel).

\section{Knockdown of ADAM8 influences proliferation and apoptosis of CRC cells}

Cell proliferation assays revealed that si-ADAM8-1mediated decreased expression of ADAM8 significantly inhibited cell proliferation in HT29 cells (down to 30.5\%; $p<0.01$ ), and si-ADAM8-2 transfection also inhibited

Table 2 Comparison of ADAM8 protein level in normal and tumor tissues by IHC $(n=42)$

\begin{tabular}{|c|c|c|c|c|}
\hline \multirow[t]{2}{*}{ Tissue } & \multicolumn{2}{|c|}{ ADAM8 expression } & \multirow[t]{2}{*}{$x^{2}$} & \multirow[t]{2}{*}{$p$ value } \\
\hline & Negative & Positive & & \\
\hline Normal & $20(66.7 \%)$ & $10(33.3 \%)$ & 13.3 & $<0.0001$ \\
\hline Tumor & 6(19.0\%) & $24(81.0 \%)$ & & \\
\hline
\end{tabular}




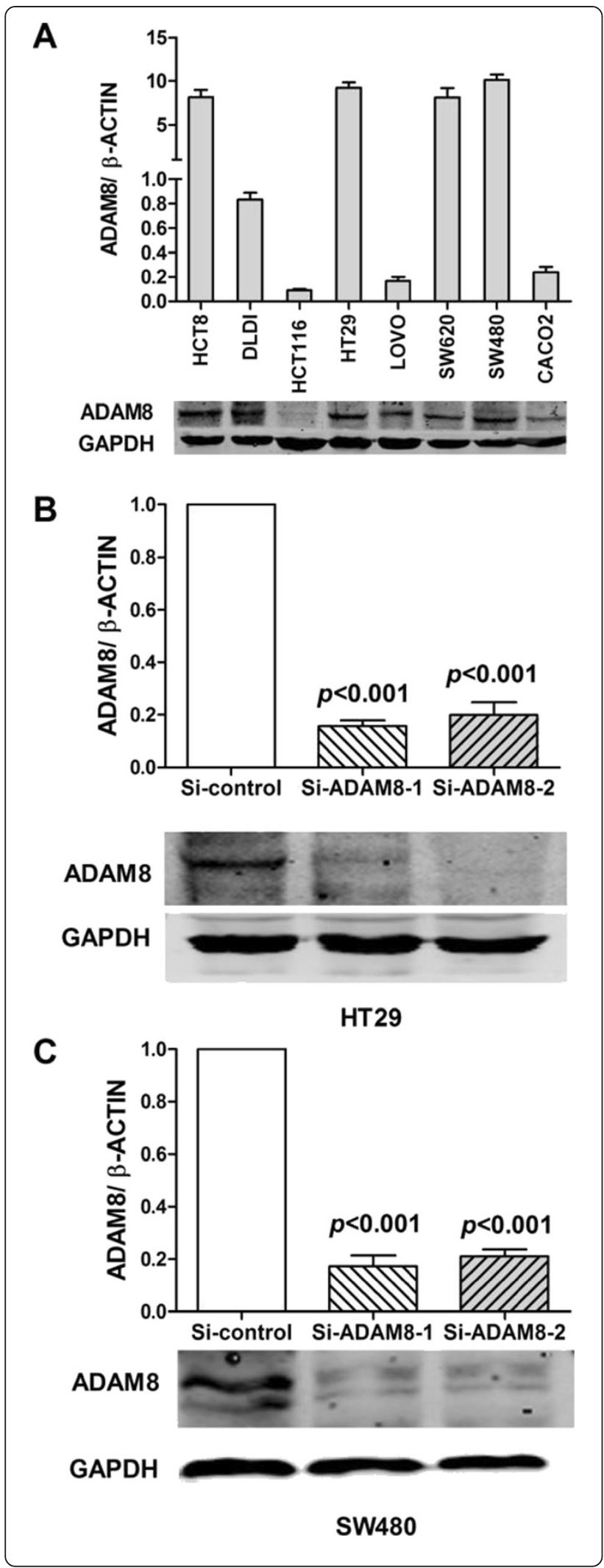

Figure 2 Expression of ADAM8 in CRC cell lines. A, Expression of ADAM8 mRNA (top) and protein level (bottom) in HCT8, HT29, SW620, SW480, DLD1, HCT116, LOVO and CACO2 cell lines. B, C, Significantly decreased expression of ADAM8 mRNA (top) and protein levels (bottom) in ADAM8 siRNA-transfected HT29 cells (B) and SW480 cells (C) compared with the control group $(p<0.001)$. The expression of ADAM8 mRNA was normalized to $\beta$-actin mRNA, which served as the control. Data are expressed as the mean \pm standard deviation (SD).

proliferation (down to 48.6\%; $p<0.001$ ) (Figure 3A). Similar results were observed in SW480 cells transfected with si-ADAM8-1 (down to 45.6\%; $p<0.001$ ) and siADAM8-2 (down to $45.2 \% ; p<0.001$ ) (Figure 3A). Cell viability assay demonstrated that knockdown of ADAM8 significantly inhibited cell growth in both SW480 (Figure 3B) and HT29 cells (Figure 3C). Cell cycle and apoptosis assay showed that the percentage of cell apoptosis in siADAM8-transfected HT29 and SW480 cells was significantly higher than that in control cells (Figure 3D). Together these results suggest that ADAM8 is involved in CRC carcinogenesis by accelerating proliferation/growth and inducing apoptosis of CRC cells.

\section{Correlation of ADAM8 expression with clinicopathological} characteristics and long-term survival of CRC

The association between ADAM8 expression and clinicopathological characteristics was assessed in 342 primary CRC patients. IHC was used to detect ADAM8 protein expression status, and tissues were scored as positive or negative as described in Materials and Methods. Among 342 CRC patients, ADAM8 was positive in 261 cases (76.3\%) and negative in 81 cases (23.7\%). However, no significant correlations were found between ADAM8 expression status and clinicopathological indicators (Table 3). The correlation of ADAM8 protein expression and postoperative survival was also evaluated. Five-year overall survival (OS) and disease-free survival (DFS) for all patients were $73 \%$ and $70 \%$, respectively. The 5 -year OS for patients with ADAM8 positive tumors was significantly poorer than those with ADAM8 negative tumors (70\% vs. $81 \%$, respectively; $p=0.037$ ) (Figure $4 \mathrm{~A}$ ). Similar results were found for the 5 -year DFS of CRC patients with positive and negative ADAM8 tumors (53\% vs. $80 \%$, respectively; $p=0.014$ ) (Figure 4B).

Regarding 5-year OS, univariate analysis indicated that $\mathrm{T}$ stage, $\mathrm{N}$ stage, $\mathrm{M}$ stage, TNM stage, differentiation grade, preoperative CEA and CA19-9 levels, and ADAM8 protein expression status were found to be prognostic factors (Table 4). However, multivariate analysis demonstrated that TNM stage, preoperative CEA levels and ADAM8 protein expression status $(\mathrm{HR}=1.943$; 95\% CI: $1.089-3.465, p=0.024)$ were independent prognostic indicators (Table 4). Further analysis for OS in each 

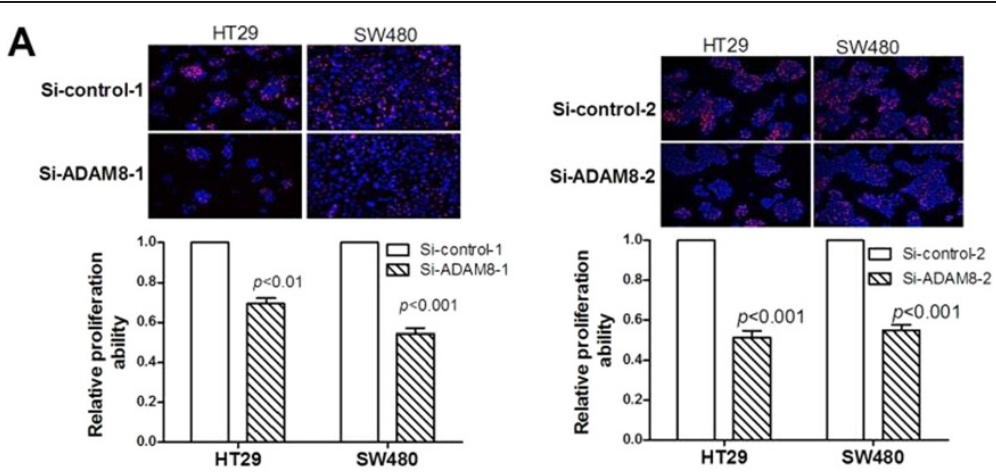

B
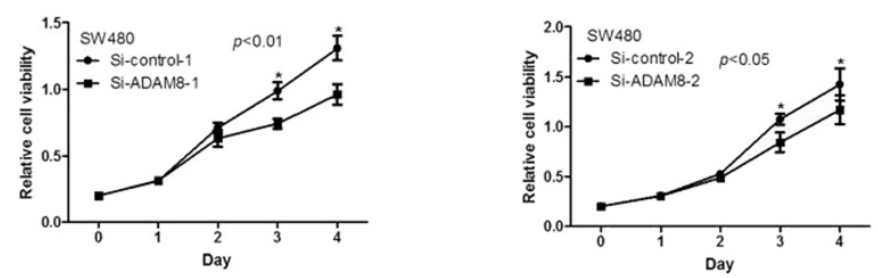

C
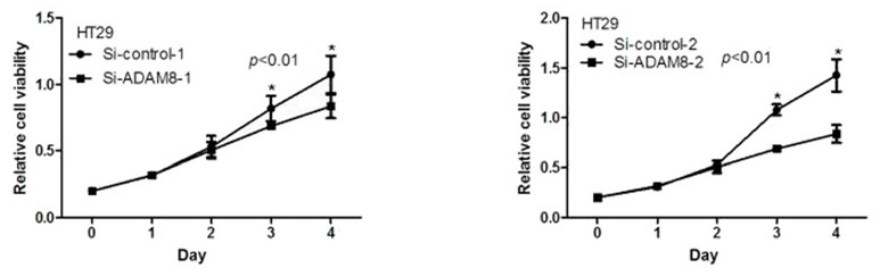

D
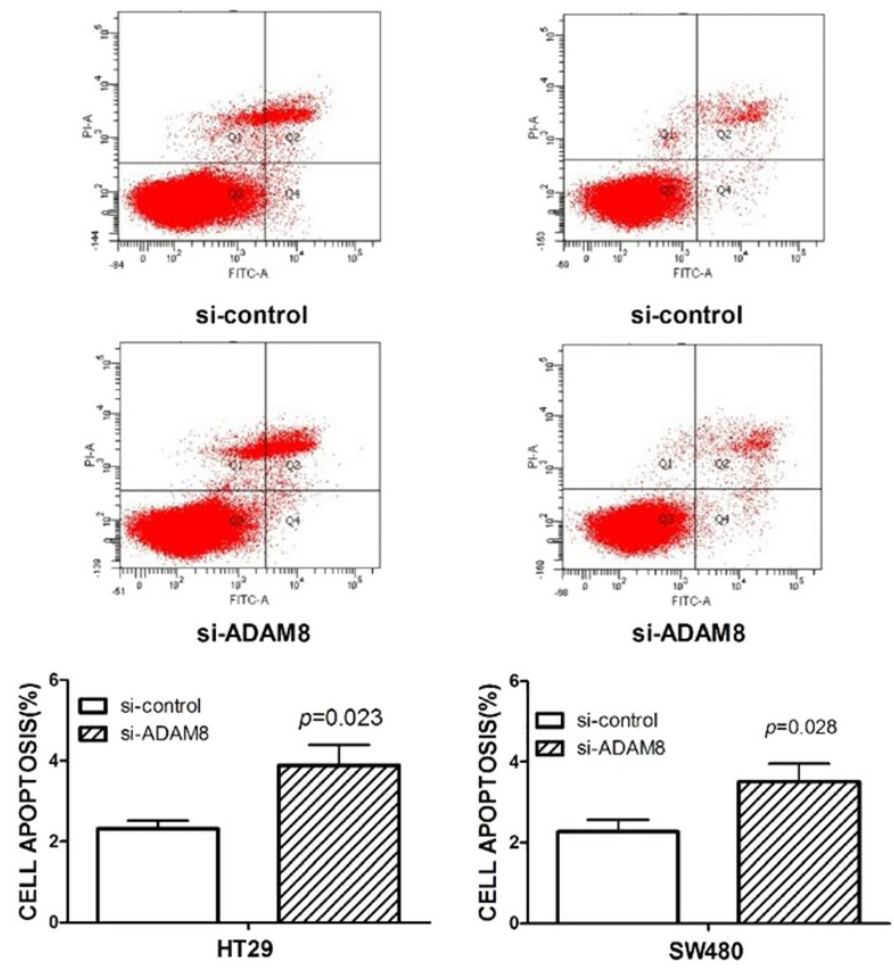

Figure 3 ADAM8 influences proliferation and apoptosis of CRC cells. ADAM8 siRNA-mediated reduction of ADAM8 significantly inhibited proliferation (A) and growth (B and C) of HT29 and SW480 cells compared with the control group. D, Knockdown of ADAM8 significantly induced cell apoptosis in HT29 and SW480 cells compared with controls. 
Table 3 Association between ADAM8 expression in CRC and clinicopathologic characteristics $(n=342)$

\begin{tabular}{|c|c|c|c|c|}
\hline \multirow[t]{2}{*}{ Indicator } & \multicolumn{2}{|c|}{ ADAM8 expression } & \multirow[t]{2}{*}{$x^{2}$} & \multirow{2}{*}{$\begin{array}{l}p \\
\text { value }\end{array}$} \\
\hline & Negative $(n=81)$ & Positive $(n=261)$ & & \\
\hline Gender & & & 1.448 & 0.229 \\
\hline Male & $49(60.5 \%)$ & $138(52.9 \%)$ & & \\
\hline Female & $32(39.5 \%)$ & $123(47.1 \%)$ & & \\
\hline Age & & & 0.769 & 0.380 \\
\hline$\leq 65 \mathrm{yrs}$ & $51(63.0 \%)$ & $150(57.5 \%)$ & & \\
\hline$>65 \mathrm{yrs}$ & $30(37.0 \%)$ & $111(42.5 \%)$ & & \\
\hline Location & & & 0.646 & 0.421 \\
\hline Colon & $42(52.9 \%)$ & $122(46.7 \%)$ & & \\
\hline Rectum & 39(48.1\%) & 139(53.3\%) & & \\
\hline T stage & & & 0.465 & 0.495 \\
\hline $\mathrm{T} 1-\mathrm{T} 2$ & $16(19.8 \%)$ & $43(16.5 \%)$ & & \\
\hline T3- T4 & $65(80.2 \%)$ & $218(83.5 \%)$ & & \\
\hline N stage & & & 1.675 & 0.196 \\
\hline No & $54(66.7 \%)$ & 153(58.7\%) & & \\
\hline N1- N2 & $27(33.3 \%)$ & $108(41.3 \%)$ & & \\
\hline M stage & & & 0.256 & 0.613 \\
\hline MO & $68(84.0 \%)$ & $225(86.2 \%)$ & & \\
\hline M1 & 13(16.0\%) & $36(13.8 \%)$ & & \\
\hline TNM stage & & & 0.544 & 0.909 \\
\hline I & $8(9.9 \%)$ & $33(12.6 \%)$ & & \\
\hline$\|$ & $33(40.7 \%)$ & $107(41.0 \%)$ & & \\
\hline III & $28(34.6 \%)$ & $83(31.8 \%)$ & & \\
\hline IV & $12(14.8 \%)$ & $38(14.6 \%)$ & & \\
\hline Differentiation grade & & & 2.863 & 0.239 \\
\hline Well & $5(12.3 \%)$ & $25(7.8 \%)$ & & \\
\hline Moderately & $71(75.4 \%)$ & $207(83.0 \%)$ & & \\
\hline Poorly & $5(12.3 \%)$ & $29(9.2 \%)$ & & \\
\hline Histological type & & & 1.209 & 0.272 \\
\hline Adenocarcinoma & $72(88.9 \%)$ & 219(83.9\%) & & \\
\hline Mucinous/Signet-ring adenocarcinoma & $9(11.1 \%)$ & $42(16.1 \%)$ & & \\
\hline CA19-9 (ug/L) & & & 3.582 & 0.058 \\
\hline$\leq 60$ & $67(82.2 \%)$ & $236(90.4 \%)$ & & \\
\hline$>60$ & $14(17.8 \%)$ & $25(9.6 \%)$ & & \\
\hline CEA (ug/L) & & & 0.225 & 0.635 \\
\hline$\leq 5$ & $52(64.2 \%)$ & $175(67.0 \%)$ & & \\
\hline$>5$ & $29(35.8 \%)$ & $86(33.0 \%)$ & & \\
\hline
\end{tabular}

subgroup showed that patients with ADAM8 positive tumors have poorer 5-year OS than those with negative ADAM8 in colon cancer $(p=0.006)$ (Figure $5 \mathrm{~A}), \mathrm{T} 3 / \mathrm{T} 4$ stage $(p=0.023)$ (Figure $5 \mathrm{~B})$ and N0 stage $(p=0.032)$ (Figure 5C) subgroups compared with rectal cancer, T1/T2 stage and N1-2 stage patients. No significant correlation was found with other subgroups.
Regarding 5-year DFS, N stage, differentiation grade, TNM stage, preoperative CEA and CA19-9 levels, and ADAM8 protein expression status were found to be prognostic factors by univariate analysis (Table 5). However, TNM stage, preoperative CEA levels and ADAM8 protein expression status $(\mathrm{HR}=2.108 ; 95 \%$ CI: $1.922-3.480, p=0.025)$ were independent prognostic 

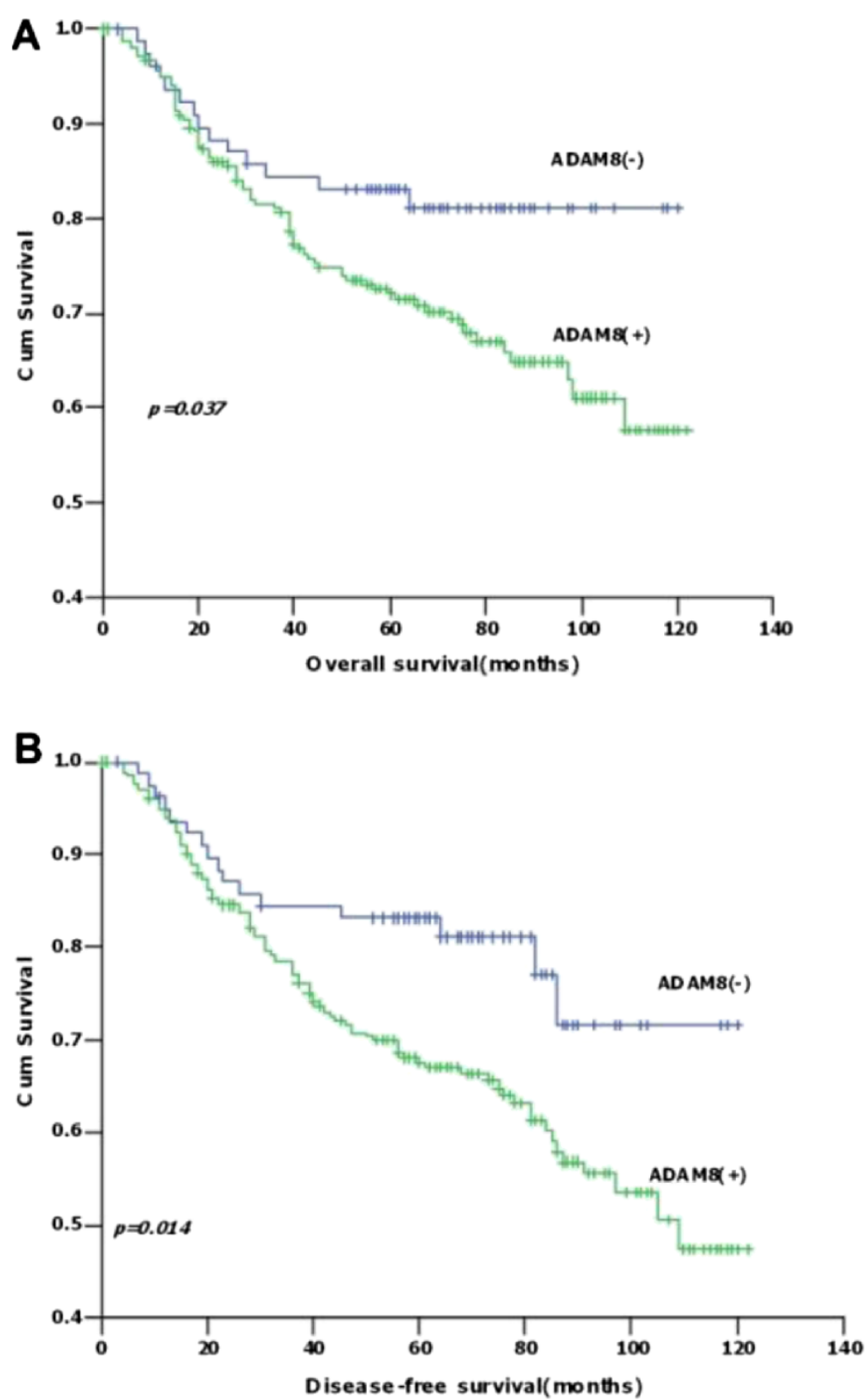

Figure 4 Survival of CRC patients according to the expression status of ADAM8 protein. A, CRC patients with positive ADAM8 had poorer OS than those with negative ADAM8 $(p=0.037)$. B, CRC patients with positive ADAM8 had poorer DFS than those with negative $\operatorname{ADAM8}(p=0.014)$.

indicators by multivariate analysis (Table 5). Further analysis for DFS in subgroups showed that patients with positive ADAM8 expression had poorer 5-year DFS than those with negative ADAM8 expression in colon cancer $(p=0.001)$ (Figure 6A), T3/T4 stage $(p=0.009)$ (Figure 6B), N0 stage $(p=0.010)$ (Figure 6C), TNM II stage $(p=0.045)$ (Figure $6 \mathrm{D})$, adenocarcinoma $(p=$ $0.027)$ (Figure 6E), moderate differentiation $(p=0.043)$ (Figure 6F) and male CRC patients $(p=0.030$ ) (Figure 6G) subgroups. No significant correlations were found with other indicators.

\section{Discussion}

A member of the ADAM family, ADAM8 has been detected in many cell types and various types of cancer [12,25-30]. However, no study of ADAM8 expression in CRC patients has been performed. In the present study, expression of both protein and mRNA levels of ADAM8 in 30 CRC patients were significantly higher in cancerous tissues than corresponding adjacent normal tissues, suggesting its importance in CRC carcinogenesis. IHC analysis of 342 CRC patients identified 261 (76.3\%) cases with positive ADAM8 expression and 81 (23.7\%) 
Table 4 Univariate and multivariate analyses of the prognostic factors for 5 -year OS of CRC patients $(n=342)$

\begin{tabular}{|c|c|c|c|c|c|}
\hline \multirow[t]{2}{*}{ Indicator } & \multicolumn{2}{|c|}{ Univariate analysis } & \multicolumn{3}{|c|}{ Multivariate analysis } \\
\hline & 5-year OS & $p$ value & $\mathrm{HR}$ & $95 \% \mathrm{Cl}$ & $p$ value \\
\hline \multicolumn{6}{|l|}{ T stage } \\
\hline $\mathrm{T} 1-\mathrm{T} 2$ & $79 \%$ & 0.009 & & & NS \\
\hline T3-T4 & $64 \%$ & & & & \\
\hline \multicolumn{6}{|l|}{$\mathrm{N}$ stage } \\
\hline No & $78 \%$ & $<0.0001$ & & & NS \\
\hline $\mathrm{N} 1-\mathrm{N} 2$ & $61 \%$ & & & & \\
\hline \multicolumn{6}{|l|}{ M stage } \\
\hline Mo & $73 \%$ & $<0.0001$ & & & NS \\
\hline M1 & $23 \%$ & & & & \\
\hline \multicolumn{6}{|c|}{ Differentiation grade } \\
\hline Well & $74 \%$ & 0.004 & & & NS \\
\hline Moderately & $72 \%$ & & & & \\
\hline Poorly & $47 \%$ & & & & \\
\hline \multicolumn{6}{|l|}{ TNM stage } \\
\hline 1 & $86 \%$ & $<0.0001$ & 1 & Reference & $<0.0001$ \\
\hline$\|$ & $78 \%$ & & 1.016 & $0.411-2.511$ & \\
\hline III & $69 \%$ & & 1.674 & $0.686-4.083$ & \\
\hline IV & $22 \%$ & & 9.685 & $3.773-24.862$ & \\
\hline \multicolumn{6}{|l|}{ CEA(ug/L) } \\
\hline$\leq 5$ & $80 \%$ & $<0.0001$ & 1 & Reference & 0.006 \\
\hline$>5$ & $54 \%$ & & 1.144 & $0.465-2.811$ & \\
\hline CA19-9 (ug/L) & & 0.040 & & & \\
\hline$\leq 60$ & $72 \%$ & & & & NS \\
\hline$>60$ & $59 \%$ & & & & \\
\hline \multicolumn{6}{|c|}{ ADAM8 protein } \\
\hline Negative & $81 \%$ & 0.037 & 1 & Reference & 0.024 \\
\hline Positive & $67 \%$ & & 1.943 & $1.089-3.465$ & \\
\hline
\end{tabular}

$\mathrm{CRC}$, colorectal cancer; $\mathrm{HR}$, hazard ratio; $\mathrm{Cl}$, confidence interval; $\mathrm{NS}$, not significant.

with negative ADAM8 expression, indicating that ADAM8 is upregulated in human CRC. To explore the potential role of ADAM8 in CRC carcinogenesis, cell proliferation and apoptosis assay were used to assess the influence of ADAM8 on cell growth. Our findings showed that siRNA-mediated downregulation of ADAM8 in CRC cells significantly suppressed cell proliferation and induced cell apoptosis, which is in agreement with previous reports $[13,16]$. These data strongly suggest that ADAM8 is involved in CRC carcinogenesis and regulates cell growth by accelerating cell proliferation and inhibiting cell apoptosis. Although previous studies have shown that ADAM8 increases invasion and migration abilities of tumor cells [14,15,21], we did not find a significant decrease of invasion and migration in ADAM8 siRNA-transfected cells compared with control cells (data not shown).
In the present study, we explored the relationship between ADAM8 expression status and clinicopathological features in CRC. Although previous studies reported that ADAM8 expression correlates significantly with tumor size, histological differentiation, regional and distant metastasis, tumor stages in several cancers progression $[12,13,29,31]$, we did not find any significant correlations between ADAM8 expression status and any clinicopathological feature in CRC.

In the present study, patients with ADAM8 positive tumors have poorer 5-year OS and DFS than those with ADAM8 negative tumors. Multivariate analysis revealed that ADAM8 positive expression could act as an important factor for unfavorable prognosis in both OS and DFS for CRC patients independent of some conventional indicators, which is in agreement with published papers $[25,29,30]$. Further analysis of survival 


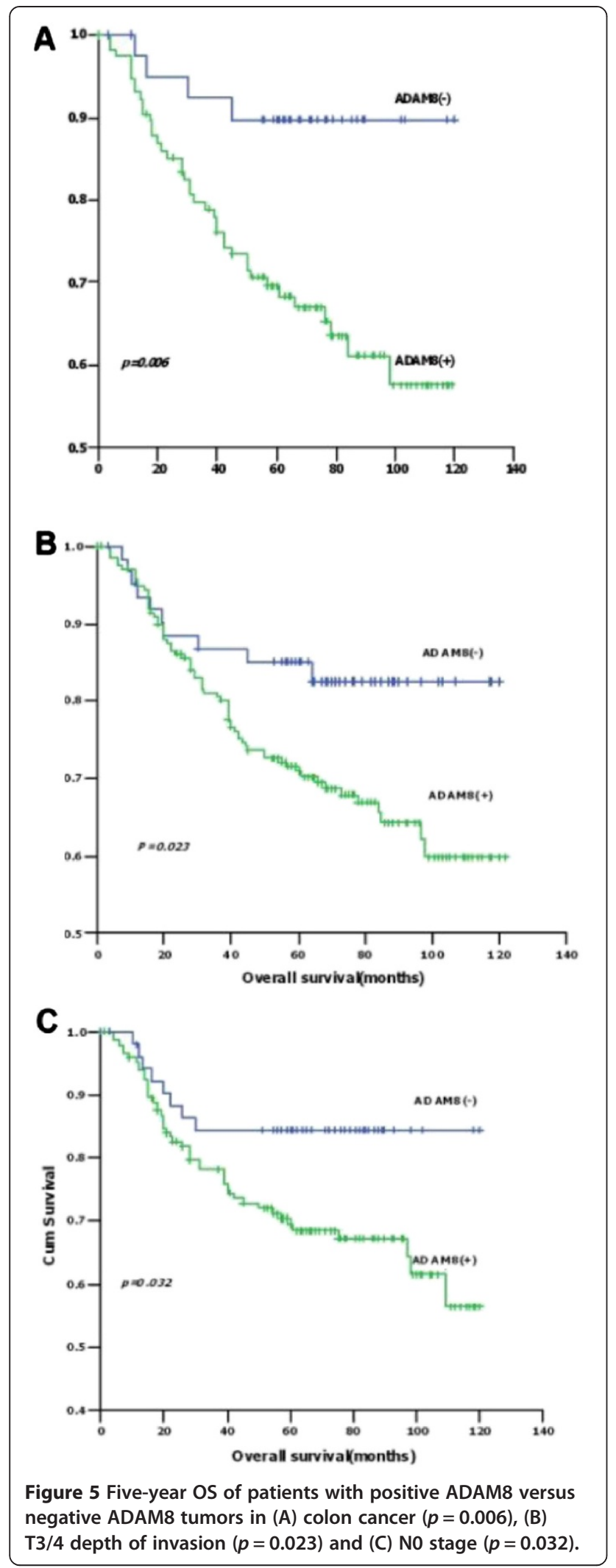

Table 5 Univariate and multivariate analyses of the prognostic factors for 5-year DFS of CRC patients $(n=292)$

\begin{tabular}{lllllll}
\hline Indicator & \multicolumn{2}{l}{ Univariate analysis } & & \multicolumn{2}{l}{ Multivariate analysis } \\
\cline { 2 - 3 } \cline { 5 - 7 } & 5 -year DFS & $p$ value & & HR & $95 \% \mathrm{Cl}$ & $p$ value \\
\hline
\end{tabular}

$\mathrm{N}$ stage

$\begin{array}{llll}\text { No } & 68 \% & 0.031 & \text { NS }\end{array}$

N1-N2 $\quad 59 \%$

Grade

$\begin{array}{llll}\text { Well } & 71 \% & 0.003 & \text { NS } \\ \text { Moderately } & 57 \% & & \\ \text { Poorly } & 46 \% & & \end{array}$

TNM stage

$\begin{array}{llllll}\text { I } & 85 \% & 0.017 & 1 & \text { Reference } & 0.027 \\ \text { II } & 72 \% & & 2.445 & 0.958-6.236 & \\ \text { III } & 61 \% & & 2.482 & 0.954-6.457 & \end{array}$

CEA(ug/L)

$\begin{array}{llllll}\leq 5 & 75 \% & <0.0001 & 1 & \text { Reference } & 0.001 \\ >5 & 52 \% & & 2.052 & 1.342-3.145 & \end{array}$

CA19-9 (ug/L)

$\begin{array}{llll}\leq 60 & 69 \% & 0.016 & \text { NS } \\ >60 & 55 \% & & \end{array}$

ADAM8 protein

$\begin{array}{llllll}\text { Negative } & 81 \% & 0.014 & 1 & \text { Reference } & 0.025 \\ \text { Positive } & 67 \% & & 2.108 & 1.922-3.480 & \end{array}$

$\mathrm{CRC}$, colorectal cancer; $\mathrm{HR}$, hazard ratio; $\mathrm{Cl}$, confidence interval; $\mathrm{NS}$, not significant.

in patient subgroups suggested that ADAM8 is a prognostic factor for colon cancer but not for rectal cancer, indicating that ADAM8 may not function as a biomarker for rectal cancer. Meanwhile, positive ADAM8 was an adverse indicator for both OS and DFS in T3/T4 depth of invasion and $\mathrm{N}_{0}$ stage, and only for DFS in adenocarcinoma, moderately differentiated tumors and male patients. Based on these results, ADAM8 can be considered as a novel prognostic marker for CRC and may serve as a target for individual therapy for certain CRC patients.

Although we explored the expression status, potential roles and clinical implications of ADAM8 in CRC, the underlying mechanism by which ADAM8 influences tumor cell growth and postoperative survival of CRC patients was not investigated in this study. Furthermore, although high expression of ADAM8 induces tumor cell resistance to chemotherapy [16], we were unable to assess the role of post-operative adjuvant chemotherapy with regard to DFS and OS in context of ADAM8 expression in univariate and multivariate analyses due to the shortage of post-operative adjuvant chemotherapy data for 342 CRC patients in this study. More studies investigating these questions should be performed in the future. 
A

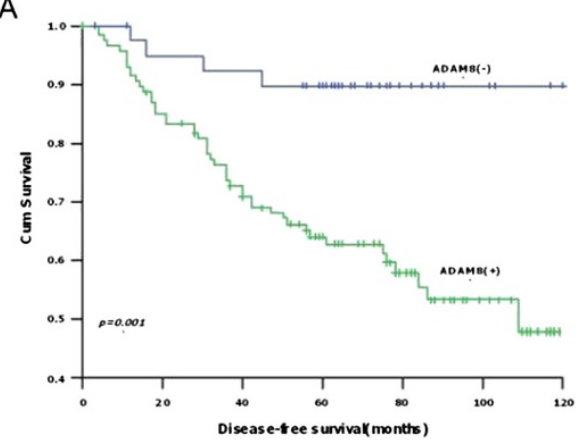

C

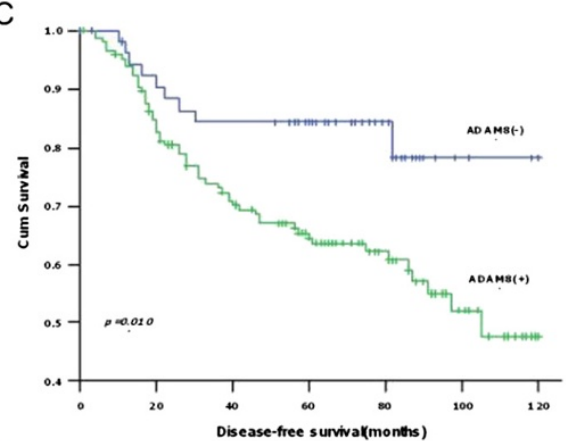

E

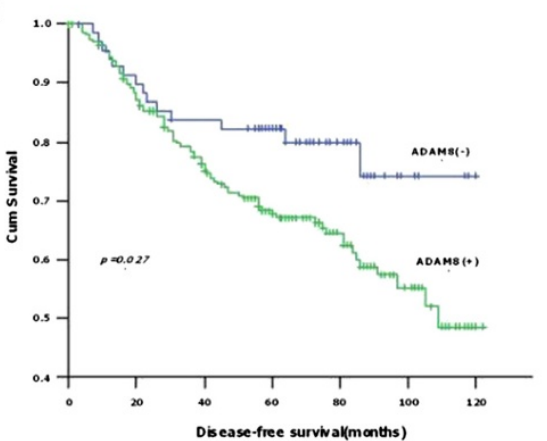

G

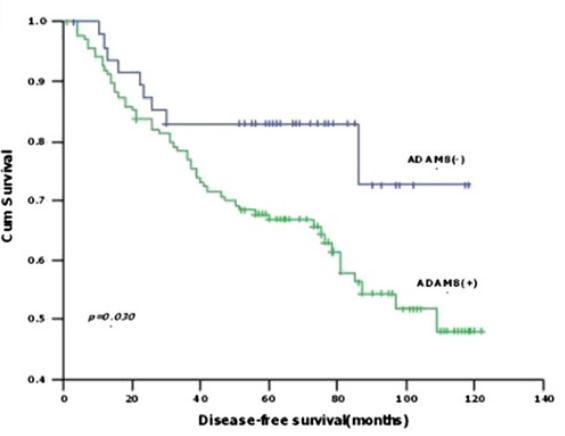

B

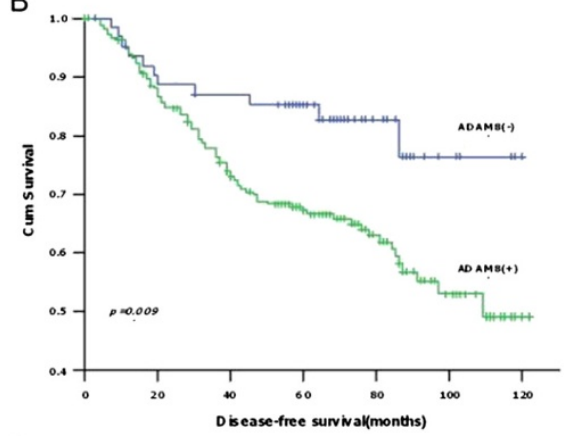

D

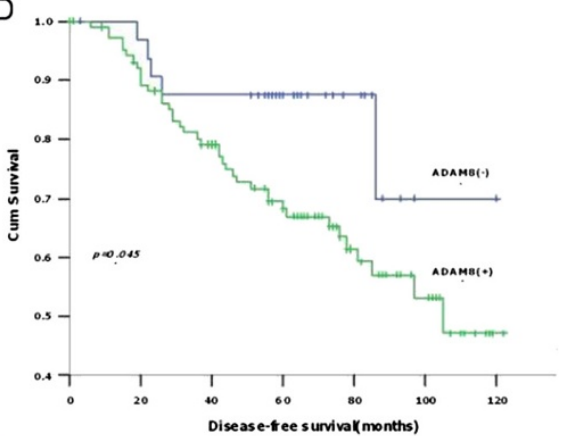

F

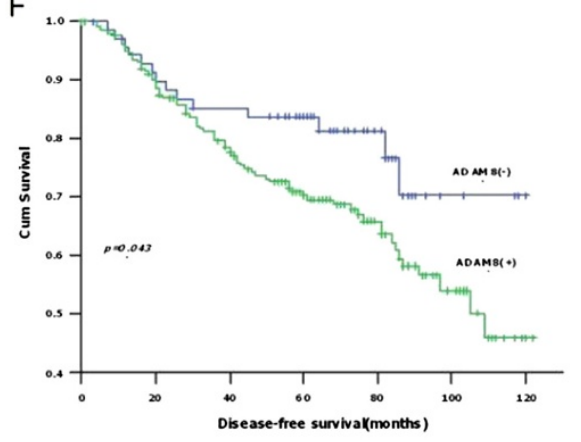

Figure 6 Five-year DFS of patients with positive ADAM8 versus negative ADAM8 tumors in (A) colon cancer $(p=0.001)$, (B) T3/4 stage $(p=0.009)$, (C) N0 stage $(p=0.032)$, (D) TNM II stage $(p=0.045)$, (E) adenocarcinoma $(p=0.027)$, (F) moderate differentiation $(p=0.043)$ and (G) male CRC patients $(p=0.030)$.

\section{Conclusions}

In summary, our results show that ADAM8 is overexpressed in CRC tissues, promoting cancer cell proliferation, inducing cell apoptosis and correlating with worse OS and DFS. Furthermore, ADAM8 may be considered as a novel prognostic marker for CRC and could function as a target of individual therapy for certain CRC patients.

\section{Abbreviations}

ADAM8: A disintegrin and metalloprotease 8; CRC: Colorectal cancer;

OS: Overall survival; DFS: Disease-free survival; qRT-PCR: Quantitative RT-PCR. 


\section{Competing interests}

The authors declare that they have no competing interests.

\section{Authors' contributions}

YB and ZLY performed most of the study and statistical analyses; LJH, HC and JZL performed part of $\mathrm{HC}$ staining; XJF and $\mathrm{JH}$ collected the clinical data and performed part of the statistical analyses; ZHY and JTH prepared some data; and JPW and LW designed the project. All authors read and approved the final manuscript.

\section{Acknowledgements}

This study was partly supported by grants from the Natural Science Fund Committee of Guangdong province (No. 10251008901000008) and the Science and Technology Planning Project of Guangdong Province (No. 2013B031600171), China.

\section{Author details}

${ }^{1}$ Department of Gastrointestinal Surgery, The Sixth Affiliated Hospital of Sun Yat-sen University (Guangdong Gastrointestinal and Anal Hospital), Sun Yat-Sen University Guangzhou, Guangzhou, P.R. China. ${ }^{2}$ Department of Ophthalmology, The First Affiliated Hospital of Sun Yat-sen University, Sun Yat-Sen University Guangzhou, Guangzhou, P.R. China. ${ }^{3}$ Gastrointestinal Institute, Sun Yat-Sen University Guangzhou, Guangzhou, P.R. China. ${ }^{4}$ Department of Colon \& Rectum Surgery, The Sixth Affiliated Hospital of Sun Yat-sen University (Guangdong Gastrointestinal and Anal Hospital), Sun Yat-Sen University Guangzhou, 26 YuancunErheng Road, Guangzhou 510655, P.R China.

Received: 12 November 2013 Accepted: 30 July 2014 Published: 7 August 2014

\section{References}

1. Yang ZL, Wang JP, Wang L, Dong WG, Huang YH, Qin JZ, Zhan WH: Multivariate regression analysis of prognostic factors in colorectal cancer. Chin-Ger J Clin Oncol 2003, 2(3):44-51.

2. Popat S, Hubner R, Houlston RS: Systematic review of micro-satellite instability and colorectal cancer prognosis. J Clin Oncol 2005, 23:609-618.

3. Kelley RK, Wang G, Venook AP: Biomarker Use in Colorectal Cancer Therapy. J Natl Compr Canc Netw 2011, 9(11):1293-1302.

4. Yokota T: Are KRAS/BRAF mutations potent prognostic and/or predictive biomarkers in colorectal cancers? Anticancer Agents Med Chem 2012, 12(2):163-171

5. Zlobec I, Bihl MP, Schwarb H, Terracciano L, Lugli A: Clinicopathological and protein characterization of BRAF- and K-RAS-mutated colorectal cancer and implications for prognosis. Int J Cancer 2010, 127(2):367-380.

6. Schlomann U, Rathke-Hartlieb S, Yamamoto S, Jockusch H, Bartsch JW: Tumor necrosis factor alpha induces a metalloprotease-disintegrin, ADAM8 (CD156): implications for neuronglia interactions during neurodegeneration. J Neurosci 2000, 20:7964-7971.

7. Dehmel T, Janke A, Hartung HP, Goebel HH, Wiendl H, Kieseier BC: The cell-specific expression of metalloproteinase-disintegrins (ADAMs) in inflammatory myopathies. Neurobiol Dis 2007, 25:665-674.

8. Dijkstra A, Postma DS, Noordhoek JA, Lodewijk ME, Kauffman HF, ten Hacken NH, Timens W: Expression of ADAMs ("a disintegrin and metalloprotease") in the human lung. Virchows Arch 2009, 454:441-449.

9. Ishikawa N, Daigo Y, Yasui W, Inai K, Nishimura H, Tsuchiya E, Kohno N, Nakamura Y: ADAM8 as a novel serological and histochemical marker for lung cancer. Clin Cancer Res 2004, 10:8363-8370.

10. Valkovskaya N, Kayed H, Felix K, Hartmann D, Giese NA, Osinsky SP, Friess H, Kleeff J: ADAM8 expression is associated with increased invasiveness and reduced patient survival in pancreatic cancer. J Cell Mol Med 2007, 11(5):1162-1174

11. Roemer A, Schwettmann L, Jung M, Stephan C, Roigas J, Kristiansen G, Loening SA, Lichtinghagen $R$, Jung $K$ : The membrane proteases adams and hepsin are differentially expressed in renal cell carcinoma. Are they potential tumor markers? J Urol 2004, 172:2162-2166.

12. Fritzsche FR, Jung $M, X u C$, Rabien A, Schicktanz H, Stephan C, Dietel M, Jung K, Kristiansen G: ADAM8 expression in prostate cancer is associated with parameters of unfavorable prognosis. Virchows Arch 2006, 449(6):628-636.
13. Zhang $Y$, Tan YF, Jiang C, Zhang $K$, Zha TZ, Zhang M: High ADAM8 expression is associated with poor prognosis in patients with hepatocellular carcinoma. Pathol Oncol Res 2013, 19(1):79-88.

14. Romagnoli M, Mineva ND, Polmear M, Conrad C, Srinivasan S, Loussouarn D, Barillé-Nion S, Georgakoudi I, Dagg A, McDermott EW, Duffy MJ, McGowan PM, Schlomann U, Parsons M, Bartsch JW, Sonenshein GE: ADAM8 expression in invasive breast cancer promotes tumor dissemination and metastasis. EMBO Mol Med 2014, 6(2):278-294.

15. Guaiquil VH, Swendeman S, Zhou W, Guaiquil P, Weskamp G, Bartsch JW Blobel CP: ADAM8 is a negative regulator of retinal neovascularization and of the growth of heterotopically injected tumor cells in mice. $J \mathrm{Mol}$ Med (Berl) 2010, 88(5):497-505.

16. Zhang W, Wan M, Ma L, Liu X, He J: Protective effects of ADAM8 against cisplatin-mediated apoptosis in non-small-cell lung cancer. Cell Biol Int 2013, 37(1):47-53.

17. Hirao T, Nanba D, Tanaka M, Ishiguro H, Kinugasa Y, Doki Y, Yano M, Matsuura N, Monden M, Higashiyama S: Overexpression of ADAM9 enhances growth factor-mediated recycling of E-cadherin in human colon cancer cell line HT29 cells. Exp Cell Res 2006, 312(3):331-339.

18. Gavert N, Sheffer M, Raveh S, Spaderna S, Shtutman M, Brabletz T, Barany F, Paty P, Notterman D, Domany E, Ben-Ze'ev A: Expression of L1-CAM and ADAM10 in human colon cancer cells induce metastasis. Cancer Res 2007, 67(16):7703-7712.

19. Van Schaeybroeck S, Kyula JN, Fenton A, Fenning CS, Sasazuki T, Shirasawa S, Longley DB, Johnston PG: Oncogenic Kras promotes chemotherapyinduced growth factor shedding via ADAM17. Cancer Res 2011, 71(3):1071-1080.

20. Choi JS, Kim KH, Jeon YK, Kim SH, Jang SG, Ku JL, Park JG: Promoter hypermethylation of the ADAM23 gene in colorectal cancer cell lines and cancer tissues. Int J Cancer 2009, 124(6):1258-1262.

21. Ashktorab H, Schäffer AA, Daremipouran M, Smoot DT, Lee E, Brim H: Distinct genetic alterations in colorectal cancer. PLOS One 2010, 5(1):e8879.

22. Gillett CE, Springall RJ, Barnes DM, Hanby AM: Multiple tissue core arrays in histopathology research: avalidation study. J Pathol 2000, 192:549-553.

23. Xavier LL, Viola GG, Ferraz AC, Da Cunha C, Deonizio JM, Netto CA, Achaval $\mathrm{M}$ : A simple and fast densitometric method for the analysis of tyrosine hydroxylase immunoreactivity in the substantia nigra pars compacta and in the ventral tegmental area. Brain Res Brain Res Protoc 2005, 16(1-3):58-64

24. Wu X, Zou Y, He X, Yuan R, Chen Y, Lan N, Lian L, Wang F, Fan X, Zeng Y, Ke J, Wu X, Lan P: Tumor-infiltrating mast cells in colorectal cancer as a poor prognostic factor. Int J Surg Pathol 2013, 21(2):111-120.

25. Wildeboer D, Naus S, Amy Sang QX, Bartsch JW, Pagenstecher A: Metalloproteinase disintegrins ADAM8 and ADAM19 are highly regulated in human primary brain tumors and their expression levels and activities are associated with invasiveness. J Neuropathol Exp Neurol 2006, 65:516-527.

26. Valkovskaya NV: Hypoxia-dependent expression of ADAM8 in human pancreatic cancer cell lines. Exp Oncol 2008, 30:129-132.

27. Hernández I, Moreno JL, Zandueta C, Montuenga L, Lecanda F: Novel alternatively spliced ADAM8 isoforms contribute to the aggressive bone metastatic phenotype of lung cancer. Oncogene 2010, 29:3758-3769.

28. Zielinski V, Brunner M, Heiduschka G, Schneider S, Seemann R, Erovic B, Thurnher D: ADAM8 in squamous cell carcinoma of the head and neck: a retrospective study. BMC Cancer 2012, 12:76.

29. Li Z, Liao Q, Wu Y, Liao M, Hao Y, Zhang S, Song S, Li B, Zhang YD: Upregulation of a disintegrin and metalloprotease 8 influences tumor metastasis and prognosis in patients with osteosarcoma. Pathol Oncol Res 2012, 18:657-661.

30. He S, Ding L, Cao Y, Li G, Deng J, Tu Y, Wang B: Overexpression of a disintegrin and metalloprotease 8 in human gliomas is implicated in tumor progression and prognosis. Med Oncol 2012, 29:2032-2037.

31. Li W, Ye F, Wang D, Sun X, Tong W, Lian G, Jiang J, Suo J, Zhang DY: Protein predictive signatures for lymph node metastasis of gastric cancer. Int J Cancer 2013, 132(8):1851-1859.

doi:10.1186/1471-2407-14-568

Cite this article as: Yang et al:: Expression of A disintegrin and metalloprotease 8 is associated with cell growth and poor survival in colorectal cancer. BMC Cancer 2014 14:568. 